\title{
Results of availability imposed configuration details developed for K-DEMO ${ }^{1}$
}

\author{
Tom Brown ${ }^{a^{*}}$, Peter Titus ${ }^{\mathrm{a}}$, Art Brooks ${ }^{\mathrm{a}}$, Han Zhang ${ }^{\mathrm{a}}$, Hutch Neilson ${ }^{\mathrm{a}}$, Kihak Im $^{\mathrm{b}}$, Keeman Kim ${ }^{\mathrm{b}}$ \\ ${ }^{a}$ Princeton Plasma Physics Laboratory, Princeton, NJ 08543, U.S.A \\ ${ }^{b}$ National Fusion Research Institute, Daejeon, 305-806, the Republic of Korea
}

The Korean fusion demonstration reactor (K-DEMO) has completed a two year study looking at key Tokamak components and configuration options in preparation of a conceptual design phase. A key part of a device configuration centers on defining an arrangement that enhances the ability to reach high availability values by defining design solutions that foster simplified maintenance operations. To maximize the size and minimize the number of in-vessel components enlarged TF coils were defined that incorporate a pair of windings within each coil to mitigate pressure drop issues and to reduce the cost of the coils. A semi-permanent shield structure was defined to develop labyrinth interfaces between double-null plasma contoured shield modules, provide an entity to align blanket components and provide support against disruption loads - with a load path that equilibrates blanket, TF and PF loads through a base structure. Blanket piping services and auxiliary systems that interface with in-vessel components have played a major role in defining the overall device arrangement - concept details will be presented along with general arrangement features and preliminary results obtained from disruption analysis.

Keywords: maintenance approach, disruption analysis, in-vessel arrangement, reduced part count

\section{Introduction}

$\mathrm{K}-\mathrm{DEMO}$ is a $6.8 \mathrm{-m}$ tokamak being developed to operate in a staged mission with an early plasma material interaction (PMI) and material testing phase followed by a high power stage with 200-600 MW electric power output and $70 \%$ availability. Past papers [1-6] provide the background details of K-DEMO, highlighting the device design and physics details. The high availability goal of the K-DEMO second phase sets a strong requirement for developing a machine design that supports innovative design approaches that simplifies maintenance operations. The key constituent to improve operation availability is to reduce the number of invessel components, maximizing the component size given the planned maintenance scheme. For a Tokamak operating with sixteen $\mathrm{TF}$ coils the size of the TF coil establishes the size of the in-vessel component to be maintained. A horizontal maintenance scheme was evaluated where the TF size would be as big as needed to allow removal of an equal number of in-vessel components as TF coils. However, the requirement to remove interfacing auxiliary systems, the need for a very large cask containment system to contain the in-vessel module, facility interfacing issues and issues with structural support for overturning loads - the horizontal maintenance option was rejected and a vertical maintenance approach selected $[7,8]$. Instead of sizing the TF coil based on a ripple criteria the coil size for the vertical maintenance approach was establish based on minimizing the number of in-vessel components removed, maximizing the component size. The enlarged sixteen TF coil device incorporates a graded, two winding pack design. This "graded" coil approach incorporates a high field and low field coil winding developed to offset the cost of the large TF coil and eliminate coolant pressure drop issues associated with the extended winding length of a single winding. Although the TF coil size is larger than one set by ripple alone it is substantially smaller than a TF coil sized for a horizontal maintained device.

With the enlarged TF coil, a unique in-vessel plasma segmentation approach was created that subdivides the blanket system into 48 plasma contoured "banana" shaped sub-modules: 16-inboard, 16-outboard modules located under each TF coil and 16-outboard modules located between TF coils. This design will need to be compared with ripple sized $\mathrm{TF}$ coils in vertically maintained designs that incorporate a larger number of banana shaped blanket modules $[9,10]$. A follow-on KDEMO design effort will look at a ripple sized $12 \mathrm{TF}$ arrangement of the same coil size that results in 36 blanket segments (12 inboard and 24 outboard).

Other design features have been incorporated within K-DEMO that differentiates it from alternate DEMO options currently being considered within the fusion community. Design features include the semi-permanent

\footnotetext{
* author's email: tbrown@pppl.gov

${ }^{1}$ Research supported by the National Fusion Research Institute, Daejeon, the Republic of Korea, under a research agreement with Princeton

University, and by the U.S. Department of Energy under Contract No. DE-AC02-09CH11466 with Princeton University.
} 
inboard C-shaped shield structure that supports disruption loads; provide shielding for gaps between sectors and an alignment system used for the installation of plasma components. Also, to increase maintenance access in the upper ports the major blanket piping services will be supplied from beneath the device through lower vacuum vessel vertical ports.

The development of the K-DEMO device configuration is still in progress. Vacuum vessel and blanket details, support arrangement and piping services have been defined to a point where structural evaluations can be made. The current progress of the in-vessel systems along with preliminary analysis of disruption loads and electro mechanical interaction of the ferromagnetic material comprising the blanket structure will be presented.

\section{K-DEMO design features}

\subsection{General arrangement}

The K-DEMO device reference design incorporates a double-null (DN) divertor which promotes strong plasma shaping (elongation and triangularity) forcing the divertor X-point inside the vacuum vessel, close to the plasma. The DN option promotes higher plasma performance and improved vertical position control which reduces the machine size when compared with single-null (SN) designs; however, it brings about engineering issues associated with increased number of divertor components, reduced breeding volume, added maintenance regions and services. To offset some of the negative aspects of the double null design, a different in-vessel segmentation option is being developed which has the potential to increase the TBR value and improve the maintenance of the high heat flux divertor section.

The main features of the K-DEMO reference design is shown in Figure 1. The initial concept was developed early in 2012 [1] with the two winding pack TF design introduced and adopted in mid-2013. Within this reference design the TF outer leg and vacuum vessel were shaped with large radius sections with the TF sized to accommodate radially

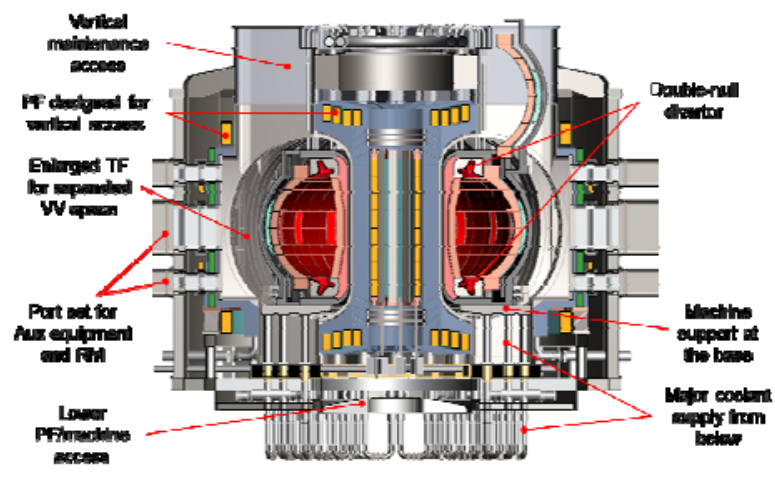

Fig. 1: K-DEMO Reference design extended vertical ports that allow removal of the formentioned 36 in-vessel blanket/shield segments and a one-sixteen segment, semi-permanent, C-shaped inner blanket support shell. The arrangement details are highlighted in Figure 2. Further shaping of the outboard region of the $\mathrm{VV}$ and $\mathrm{TF}$ has been done since the original design of the reference configuration. The outer region of the VV has been reshaped to a more oval geometry and the TF outer leg moved in about 1-m while retaining the shape of the enlarged vertical ports. The geometry changes reduced the loading on the TF inner leg and brought the outer PF ring coils closer to the plasma.

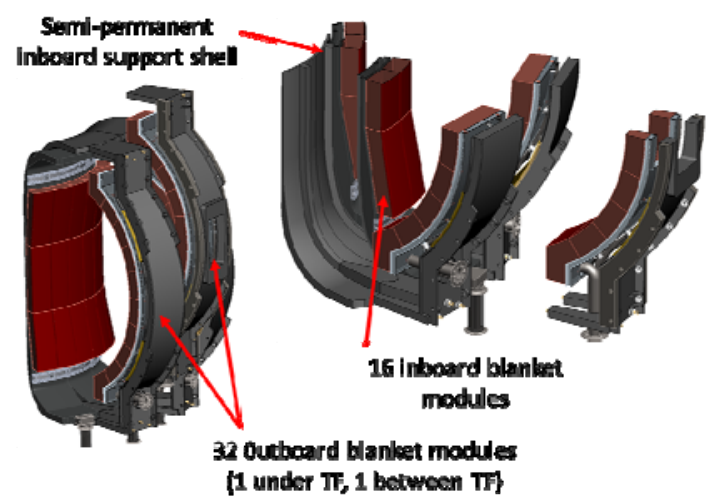

Fig. 2: K-DEMO blanket/shield segmentation

The blanket/shield system and its surrounding containment structure are attached to the base of the vacuum vessel with expected snubber connections to the TF coil at the top. Since the disruption load path is not through the vacuum vessel mid-section a lighter VV structure can be developed. Structural analysis of the reshaped vessel under vacuum loads was made with acceptable stress results (see Figure 3).

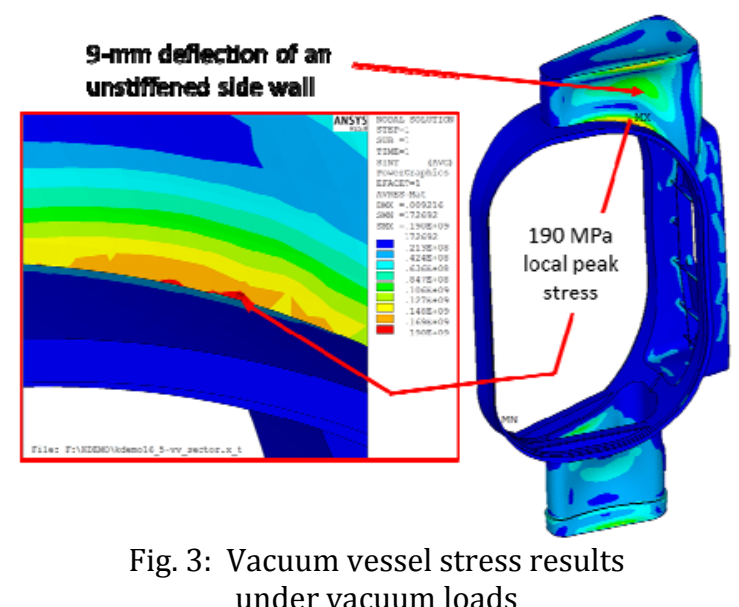

\subsection{Blanket / shield details}

Developing the functional design and analytical details of the blanket/shield component and the connecting piping services is difficult by itself but coupled with the need to arrive at a design solution that 
also meets the requirements for high operating availability presents a real engineering challenge.

Each blanket sub-module consists of tungsten plasma-facing first wall, layers of breeding parts, cooling channels, tungsten passive stabilizer. A thin layer of vanadium is placed as an interlaying material between tungsten first wall and Reduced Activation Ferritic Martensitic (RAFM) structural material. Ceramic breeder pebbles of lithium ortho-silicate (Li4SiO4) are used as a mixture with beryllide (Be12Ti) neutron multiplier pebbles. Further definition of the concept solid breeding blanket model presented in earlier documents [6] has been made with some details shown in Figure 4. Figure 4a shows the basic blanket submodule details. A $100 \mathrm{~mm}$ inlet pipe surrounded by an equal sized return pipe centrally located at the edge of cover plate. To balance the flow to the various subpanels an orifice is located on the inlet line feeding each panel. A center spline in the cover plate is used as an alignment key and internally hollowed to provide space for a large internal return duct. Attachment holes for four ITER style flexible supports are located at the corners. Local details are shown in the section views of Figure 4b. Access to the supply pipes routed within the blanket backing structure is assessable through access plates once the full blanket segment is removed from its external support structure.

Instead of employing a rectangular shaped blanket that leaves dead regions for extraction the blanket submodules were shaped as trapezoidal sections to maximize the breeding material volume. Each module is

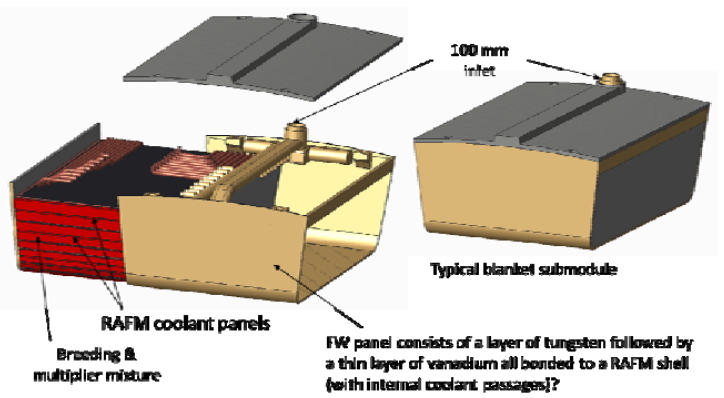

Fig. 4a: Typical blanket submodule

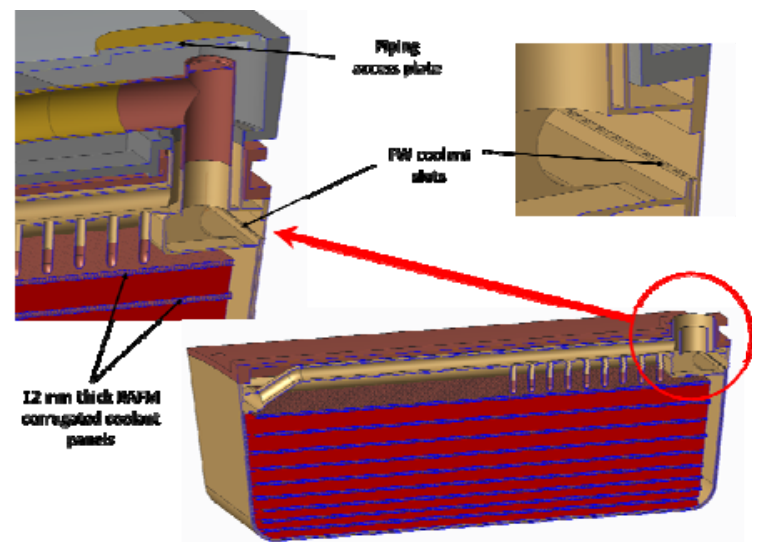

Fig. 4b: Local details shown in a section view

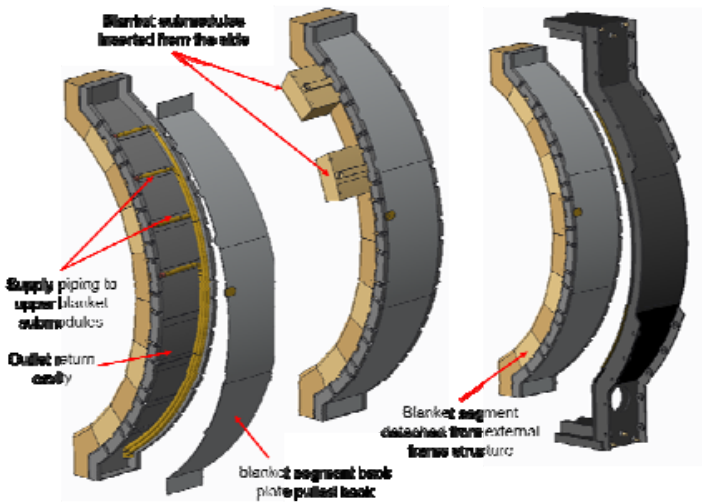

Fig. 5: Outboard blanket sector located beneath TF coils

assembled from the sides of their backing structure. The submodule supply and concentric return pipes is surrounded by a frame structure that anchors the unit at one location, with ITER style supports at the module corners to allow thermal expansion. Figure 5 shows the general arrangement for the blanket sector that is located beneath a TF coil highlighting the basic make up of a blanket sector. Although not detailed at this time a blanket segment will be attached to its external shield structure at its midplane with a number of structural support members above and below the midplane support region to allow thermal growth of the blanket sector.

The support details defined of the in-vessel blanket system is illustrated in the isometric view of Figure 6. The blanket design, supporting structure and interfacing piping services was developed to also accommodate both the solid breeding blanket and the DCLL liquid metal blanket concepts. To provide control for each blanket submodule and accommodate the MHD requirements of the DCLL, individual supply lines are routed to each module (10 blanket modules on the outside). Due to a

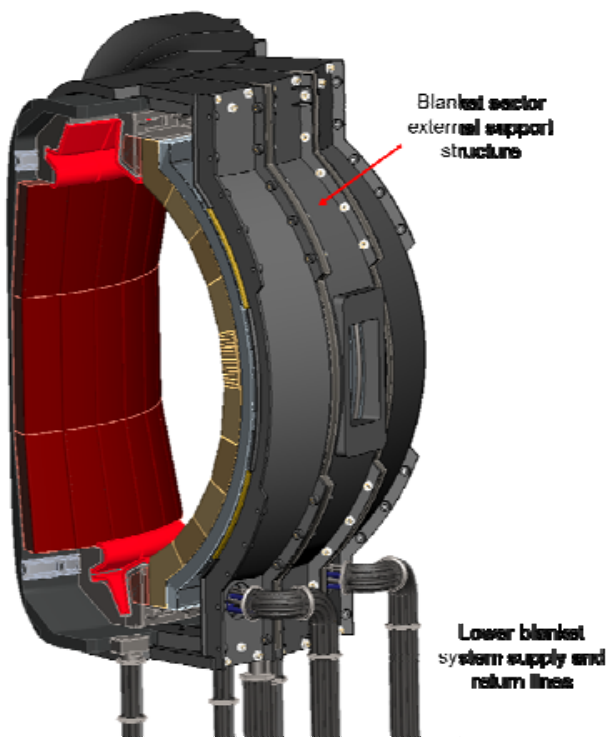

Fig. 6: In-vessel blanket support and coolant feed system 
lack of space at the outside of the blanket system, a common coolant return system is used, feeding into a single large return pipe at the bottom of the blanket sector. In order to maximize the access at the upper ports for the vertically maintained K-DEMO device the majority of the piping supplies have been located at the bottom - passing through lower vertical ports. The external blanket sector structure forms a complete shell which has been defined to support the blankets against disruption and RAFM magnetic loads.

\section{Blanket System Disruption Analysis}

Disruption analysis is intended to quantify the significant loading on the blanket structures in terms of the feasibility of various component layouts. Significant vertical loads occur on toroidally continuous structures during a Vertical Disruption Event (VDE). The planned semi-permanent blanket support structures are in this category and will require heavy column connections to ground. Another major issue is the necessity of electrically dividing up structures to minimize eddy current loads. This has been investigated by running outer segments of the blanket either poloidally connected electrically, or insulated. A major uncertainty in this evaluation is the conductivity of the breeder materials. The ceramic breeder blanket concepts include mostly non-conducting material, and the conducting material used in the structures is finely divided and kept to a minimum to maintain the breeding ratio. It is expected that with these breeder concepts the accumulation of thermal expansion differentials will be more challenging than disruption eddy currents. In the process of modeling the disruptions, the magnetic properties of the structural materials had to be addressed. Use of magnetic materials makes the analytic modeling challenging. PPPL is working on a consistent concurrent analysis of transient eddy currents in the magnetic structural materials. Two approaches using ANSYS (version 15, 2013) are being pursued. The first is a vector potential formulation with special coupling at the boundaries of the magnetic material. The second uses the edge potential element solid236. In both cases a fine mesh is required around the components for which load summations are required. A VDE has been simulated in K-DEMO with the full volume of the blanket having the magnetic properties of the RAFM steel. At this time the complexity of detailed modeling of blanket internals is too challenging, and methods to use equivalent or smeared properties will need to be developed. Modeling of magnetic materials is also being investigated using existing ITER models being used to size loads on port plugs (see Figure 7). The port plug material was changed to RAFM steel and the loading from the magnetic materials could be directly compared with loads from disruption eddy currents.

The edge potential solution was used for this evaluation. K-DEMO results for the magnetic materials showed much higher net loads than for the disruption eddy currents. The character of the loading from the magnetic materials is a direct force while the loading from the disruption eddy currents is primarily large moments.
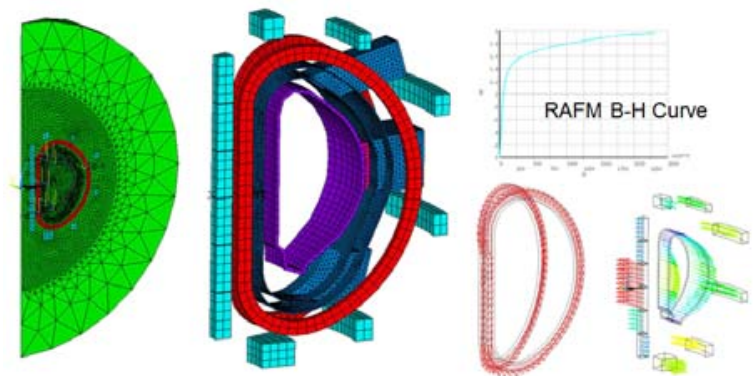

Fig. 7: Model Based on ITER used to Investigate Inclusion of Magnetic Materials in Blanket Structures

When resolved to support points, the loads from the magnetic materials with static fields applied were comparable to the loads resulting from the disruption eddy current moments. Simple hand calculations of the expected magnetic loads for the ITER (fictitious) blanket model were compared with the ANSYS results and showed good agreement. Early K-DEMO ANSYS models are shown in Figure 8.

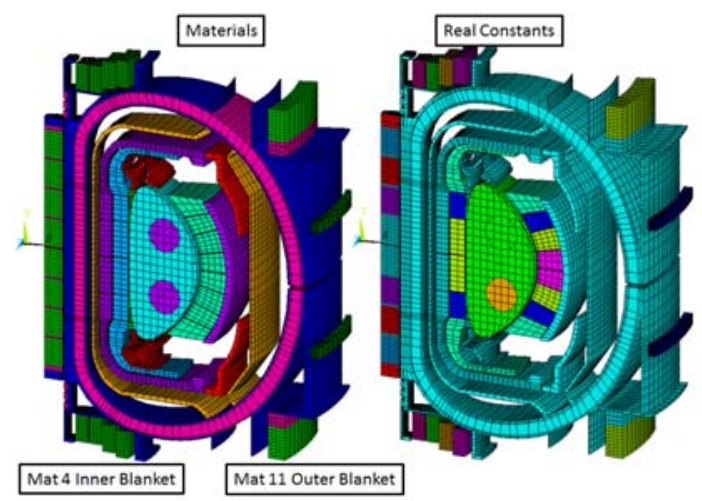

Fig. 8: Early K-DEMO Model Used in Studies of Magnetic Materials

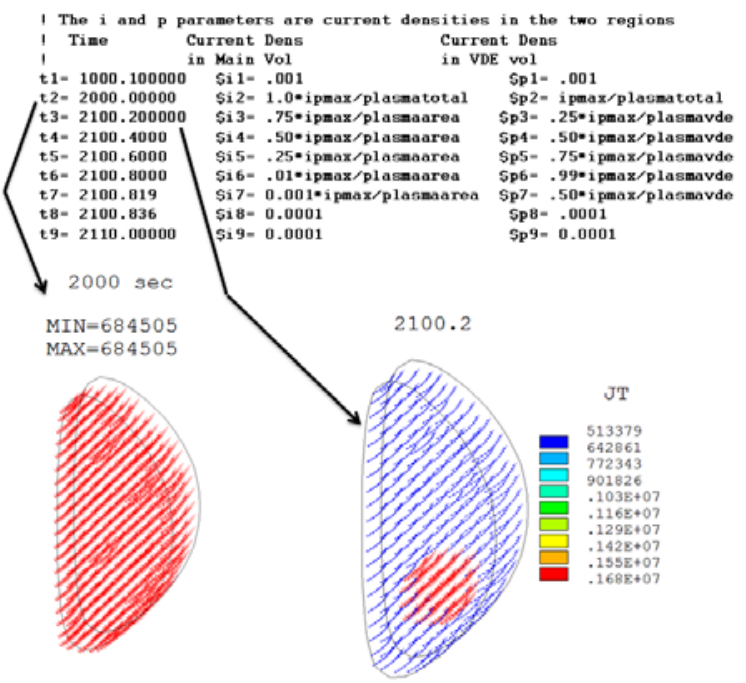

Fig. 9: Disruption Plasma Current Input Assumed for the K-DEMO Simulations 
ISFNT-12 / P0067

The VDE was chosen for K-DEMO because it potentially applied large net loads on the structures. Figure 9 shows the ANSYS ADPL input for the plasma currents vs. time. The assumed disruption specifications have been taken from ITER data and are $0.8 \mathrm{sec}$ for the drift and 36 milliseconds for the quench.

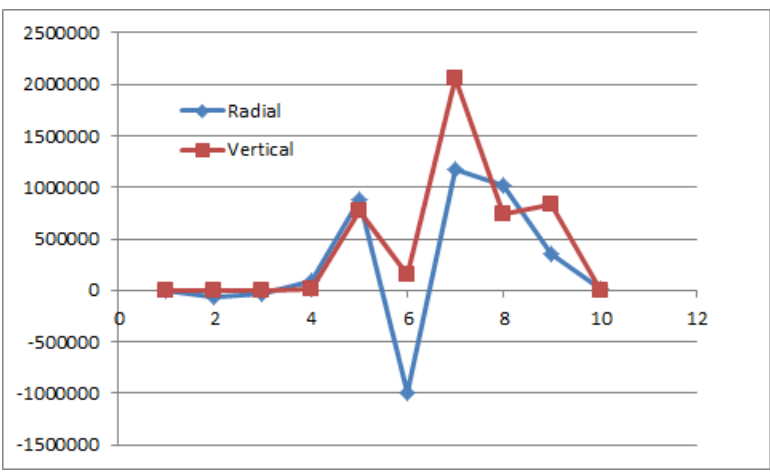

Fig. 10: K-DEMO Blanket Support Structure Loading (Newton). 1/16 sector

Figure 10 shows the net loads on the K-DEMO inner blanket support structure from the disruption eddy currents. These derive from the interaction of the toroidal currents induced from the quenching plasma interacting with the poloidal field. The large net vertical load results from the asymmetry of the VDE. Reduced activation, magnetic materials are planned for these structures and these will have a larger percentage of metal than the blankets and large - as of yet undetermined static magnetic loads.

All the disruption results produced to date are with separate modeling of magnetic materials with static fields applied, and separate modeling of the disruption transient eddy currents. Concurrent analysis is being developed. Practical modeling of blanket components will require use of "smeared" magnetic properties.

\section{Summary remarks}

The K-DEMO device configuration and supporting analysis is progressing in design and analysis detail although not yet to a level of maturity that allows full validation of design concepts that are being perused in all areas. Design and analysis details of the TF system has matured to a level that allows reasonable confidence that an acceptable solution can be realized; this also follows with the vacuum vessel design. Concept validation of the blanket system and its support arrangement is still an open question. Disruption analysis intended to quantify the significant loading on the blanket structures in terms of the feasibility of various component layouts is being made, first with understanding and validation of the analytical approach followed by analysis of the blanket system design details.

\section{References}

[1] K. Kim et al., A preliminary conceptual design study for Korean fusion Demo reactor, Fusion Engineering and Design, Volume 88, Issues 6-8, Pages 488-491, 2012.

[2] T. Brown et al., Progress in developing the K-DEMO device configuration, $25^{\text {th }}$ Symposium on Fusion Engineering, San Francisco, CA, June 2013

[3] C. Kessel et al., Systems Analysis Exploration of operating points for the Korean Demo program, $25^{\text {th }}$ Symposium on Fusion Engineering, San Francisco, CA, June 2013

[4] T. Brown et al., Availability considerations in the design of K-DEMO, $25^{\text {th }}$ IAEA Fusion Energy Conference, Oct 2014, St. Petersburg, Russia.

[5] K. Kim et al., Conceptual design study of the K-DEMO magnet system, Fusion Engineering and Design, In Press 2015.

[6] K.Kim et al., Design concept of K-DEMO for near-term implementation, Nuci. Fusion 55 (2015) 053027 (9pp)

[7] T. Brown et.al, Progress in Developing a HighAvailability Advanced Tokamak Pilot Plant, 24 ${ }^{\text {th }}$ Fusion Energy conference, San Diego, Calif., 8 October 2012.

[8] H. Utoh et al., Critical design factors for sector transport maintenance in DEMO, Nucl. Fusion 53 (2013) 123005 (8pp)

[9] A. Loving et al., Pre-conceptual design assessment of DEMO remote maintenance, Fusion Engineering and Design, Volume 89, (2014) 2246-2250.

[10] Y. Song et al., Concept design on RH maintenance of CFETR Tokamak reactor, Fusion Engineering and Design, Volume 89, Oct. 2014, Pages 2331-2335 